\title{
Phase-Shifting Transformer Combined with the Novel SHEPWM for Harmonics Reduction in Medium-Voltage Inductive Loads
}

\author{
Hussain Bierk, Fawzi Mustafa, Baraa M. Albaker
}

\begin{abstract}
In this paper, a novel approach is proposed to reduce the impact of harmonics in the high-power medium-voltage squirrel-cage induction motor. An inverter with a minimum switching frequency is required for this load type to reduce the snubber and switching losses. Phase-shifting transformer is selected as an interface between the three-phase supply and the inverter. This is to reduce the impact of low order harmonics and consequently reducing total harmonic distortion (THD). Selective harmonic-elimination pulse width modulation (SHEPWM) is employed as a control technique in the inverter to eliminate specific low-order harmonics. Thereby, many low order harmonics are reduced further. By this way the THD of the system is reduced significantly and consequently the better and cleaner energy is obtained. In this research, PSpice simulation verified by MATLAB is used to estimate the harmonics content of the currents in high-power medium voltage induction motor.
\end{abstract}

Index Terms: High-Power Induction Motor; High-Power Inverters; Low Frequency Switching Inverters; Phase-Shifting Transformers; SHEPWM.

\section{INTRODUCTION}

Generally, the challenge of harmonics reduction in power systems is adopting cost effective strategies [1]. This lead to decrease the negative impacts of harmonics in general and low-order harmonics in particular [2]. There are many techniques used to reduce the level of low-order harmonics and one of these techniques is the phase-shifting transformers. This technique results in phase-shifting of the load currents.

In this work, the technique of phase-shifting transformer is used as an interface between the power supply and three-phase inverters. These inverters are controlled by selective harmonics elimination pulse width modulation (SHEPWM). This is to eliminate some specific low-order harmonics in the load currents. The combination of these two effective methods provides, without any doubt, the novel and optimum technique to eliminate or reduce low-order harmonics in high-power inductive load.

It is well-known that there are many pulse width

Revised Manuscript Received on September 22, 2019.

Hussain Bierk, College of Engineering, Al-Iraqia University, Baghdad, Iraq, hbierk@hotmail.com

Fawzi Mustafa, College of Engineering, Al-Iraqia University, Baghdad, Iraq

Baraa M. Albaker, College of Engineering, Al-Iraqia University, Baghdad, Iraq. modulation techniques used to eliminate some specific low-order harmonics such as trapezoidal PWM, SHEPWM, and space vector modulation (SVM). Among these three methods, SHEPWM technique has the best performance in term of elimination of low-order harmonics especially in high-power induction machines with a very low switching frequency [3]. However, the only drawback of this topology is the complexity of the computation processes [4]. The principle of operation of this technique is to model the non-linear equations, which are necessary to eliminate the specific number of low-order harmonics at certain modulation index (MI). Many mathematical methods can be used effectively to solve these non-linear trigonometric equations such as Newton-Raphson method or using numerical analysis processes. The outcomes are a set or group of gating angles required by the controller for gating purpose and normally stored in look-up tables. The complexity of calculations processes depends on the number of the low-order harmonics to be eliminated. It increases with the increasing number of harmonics. Accordingly, a very large memory space is required to accommodate these pre-calculated switching angles and therefore a high intensive care is required to set-up this large amount of data.

\section{SHEPWM BASED HARMONICS REDUCTION}

Three-level SHEPWM is selected in this work for harmonics reduction and elimination. The selection is based on the fact that it possesses the better harmonics content than bi-level PWM waveform. Fig. 1 depicts a typical three-level SHEPWM to eliminate two low-order harmonics. The waveform in the figure can be analyzed by Fourier series expansion [5] as follows:

$f(\theta)=\sum_{n=1}^{\infty}\left[a_{n} \cos n \theta+b_{n} \sin n \theta\right]$

Because of the quarter-wave symmetry of this waveform, the coefficients of the above equation are as follows:

$$
\begin{aligned}
& b_{n}=\left[\begin{array}{ll}
\frac{4 V_{d c}}{n \pi} \sum_{m=1}^{N}(-1)^{m+1} \cos \left(n \alpha_{m}\right) & n \text { is odd } \\
0 & \text { n is even }
\end{array}\right. \\
& a_{n}=0
\end{aligned}
$$


where $\mathrm{n}$ represents the number of the switching angles to be calculated. From equation (2), it is clear that all even harmonics vanishes. In order to eliminate, for example, two low-order harmonics such as $5^{\text {th }}$, and $7^{\text {th }}$ harmonics, it is required to solve three nonlinear equations to calculate three switching angles $\left(\alpha_{1}, \alpha_{2}\right.$, and $\left.\alpha_{3}\right)$ as demonstrated in Fig.1.

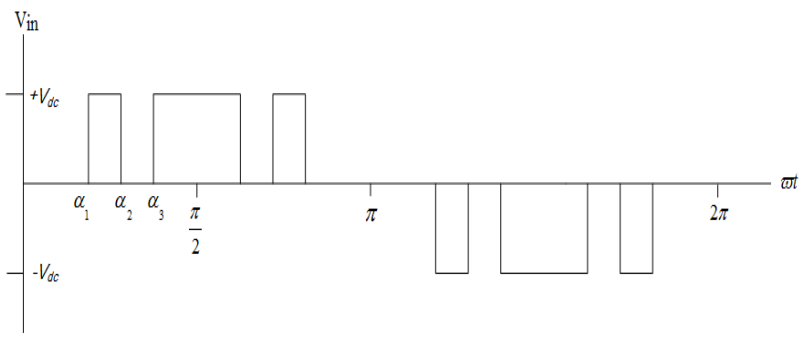

Fig. 1: SHE-PWM technique to eliminate two harmonics

To find the switching angles $\alpha_{1}, \alpha_{2}$, and $\alpha_{3}$, we have to equate $b_{1}$ (peak of the fundamental voltage normalized to $\mathrm{V}_{\mathrm{dc}}$ ) to $\mathrm{MI}$, and both $\mathrm{b}_{5}$, and $\mathrm{b}_{7}$ to zero:

$$
\begin{aligned}
& b_{1}=\frac{4}{\pi}\left(\cos \alpha_{1}-\cos \alpha_{2}+\cos \alpha_{2}\right)=\text { MI } \\
& \text { Where MI is the modulation index. } \\
& \therefore \cos \alpha_{1}-\cos \alpha_{2}+\cos \alpha_{3}=\frac{\pi}{4} \text { MI } \\
& b_{5}=\cos 5 \alpha_{1}-\cos 5 \alpha_{2}+\cos 5 \alpha_{3}=0 \\
& b_{7}=\cos 7 \alpha_{1}-\cos 7 \alpha_{2}+\cos 7 \alpha_{3}=0
\end{aligned}
$$

Then we use a numerical solver such as the Newton-Raphson method or other numerical methods to calculate these switching angles at different modulation indexes [6].

The main key to reduce the complexity of solving these equations is to reduce the number of equations that are necessary to eliminate a high number of low-order harmonics. This is done using modified SHEPWM, which is explained in the following sections.

\section{LOAD EQUIVALENT CIRCUIT}

The specifications of the used high power medium voltage induction motor (the load) are as follows:

$\begin{array}{lc}\text { Useful power per phase } & 3000 \mathrm{KW} \\ \text { Nominal phase to phase voltage: } & 3.30 \mathrm{KV} \\ \text { Nominal slip: } & 0.04 \\ \text { Full load electric efficiency : } & 0.95 \\ \text { Power factor : } & 0.90 \\ \text { Ratio of locked rotor current/ F.L current: } & 6\end{array}$

$\begin{array}{lr}\text { Ratio of locked rotor current/ F.L current: } & 6 \\ \text { Supply frequency: } & 50 \mathrm{~Hz}\end{array}$

Where:

$\mathrm{R}_{\mathrm{M}}=$ Total motor leakage resistance

$\mathrm{L}_{\mathrm{M}}=$ Total motor leakage inductance

Per phase induction motor equivalent circuit is presented in Fig. 2.

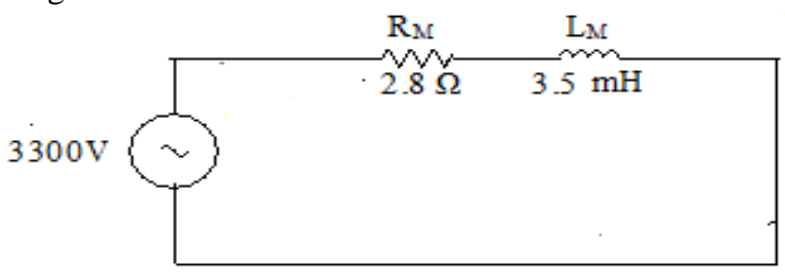

Fig. 2: Per-phase induction motor equivalent circuit

\section{TRANSFORMERLESS CONNECTION BETWEEN THEINVERTER AND LOAD}

Due to direct connection between inverter(s) and an inductive load, the common mode voltages [7] are generated during the operation of power electronic circuits especially with conversion and inversion process. The voltages are mainly zero-sequence voltages superimposed with switching noise. Basically, this phenomenon contributes to the damage of the motor windings insulation with the time and therefore it is important to mitigate or significantly reduce its adverse impacts.

\section{TRANSFORMER CONNECTION}

The method of using phase-shifting transformer as interface between the inverter and inductive load has two main advantages:

- Provides the electrical insulation between the inverter and inductive load so this avoids the premature motor winding insulation failure.

- Significantly cancels some low-order harmonics by introducing phase-shift angle between primary and secondary currents and consequently pure sinusoidal current waveform is obtained.

\section{HARMONIC CURRENTS PHASE-DISPLACEMENT}

In Figure (3) below, the phase-shifting transformer delta-connected primary side is connected to the main source. The star-connected secondary side is connected to the inductive load and based on this topology a phase-shift of harmonic currents is obtained. This phase-shift contributes to cancel some low-order harmonics which are generated by non-linear loads (such as inverters, inductive loads...etc.).

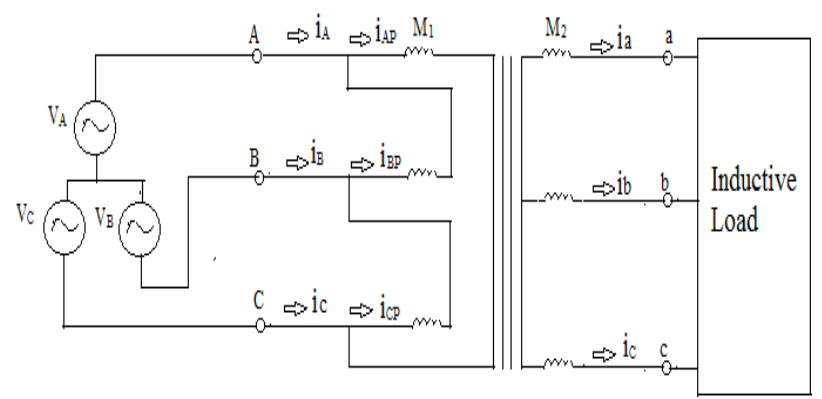

Fig. 3: Three-phase shifting transformer $\Delta / \mathrm{Y}$ connected.

If the number of the transformer primary winding turns is equal to $M_{1}$ and secondary winding turns is equal to $M_{2}$, and the voltage ratio $V_{A B} / V_{a b}$ is unity, then the turn ratio $M_{1} / M_{2}$ equals $\sqrt{3}$. The phase angle( $\varnothing)$ between the primary side of the transformer and the secondary side is $30^{\circ}$. Assuming balanced system, the line currents of the secondary of the transformer (load side) can be expressed as follows:

$$
\begin{aligned}
& \mathrm{i}_{\mathrm{a}}=\sum_{n=15,7,11}^{\infty} \mathrm{I}_{\mathrm{nM}} \sin (\mathrm{n} \omega t) \\
& \mathrm{i}_{\mathrm{b}}=\sum_{\mathrm{n}=1,5,7,11}^{\infty} \mathrm{I}_{\mathrm{nM}} \sin \left(\mathrm{n} \omega t-120^{\circ}\right) \\
& \mathrm{i}_{\mathrm{c}}=\sum_{\mathrm{n}=1.5,7,11}^{\mathrm{m}} \mathrm{I}_{\mathrm{nM}} \sin \left(\mathrm{n} \omega t-240^{\circ}\right)
\end{aligned}
$$


where $I_{n M}$ is the peak value of the nth order harmonic current. The primary currents $i_{A M}$ and $i_{B M}$ relates to $i_{a}$ and $i_{b}$ as follows:

$\mathrm{i}_{\mathrm{AM}}=\frac{\mathrm{MI}}{\mathrm{MI}} \mathrm{i}_{\mathrm{a}}=\frac{\mathrm{I}}{\mathrm{x}}\left(\mathrm{I}_{1 \mathrm{M}} \sin (\omega \mathrm{t})+\mathrm{I}_{5 \mathrm{M}} \sin (5 \omega \mathrm{t})+\mathrm{I}_{7 \mathrm{M}} \sin (7 \omega \mathrm{t})+\right.$ $\mathrm{I}_{11 \mathrm{M}} \sin (11 \omega \mathrm{t})+$.)

Similarly,

$\mathrm{i}_{\mathrm{BM}}=\frac{\mathrm{MA}}{\mathrm{MA}} \mathrm{i}_{\mathrm{b}}=\frac{1}{\mathrm{~S}}\left(\mathrm{I}_{1 \mathrm{M}} \sin \left(\omega \mathrm{t}-120^{\circ}\right)+\mathrm{I}_{5 \mathrm{M}} \sin \left(5 \omega \mathrm{t}-240^{\circ}\right)+\right.$

$I_{7 M} \sin \left(7 \omega t-120^{\circ}\right)+I_{11 M} \sin \left(11 \omega t-240^{\circ}\right)+$. $)$

The primary line current of phase $A$ can be found:

$\mathrm{I}_{\mathrm{A}=} \mathrm{i}_{\mathrm{AM}}+\mathrm{i}_{\mathrm{BM}}=\left(\mathrm{I}_{1 \mathrm{M}} \sin \left(\omega \mathrm{t}+30^{\circ}\right)+\mathrm{I}_{5 \mathrm{M}}\left(5 \omega \mathrm{t}-30^{\circ}\right)+\mathrm{I}_{7 \mathrm{M}}\right.$ $\left.\sin \left(7 \omega t+30^{\circ}\right)+I_{11 M} \sin \left(11 \omega t-30^{\circ}\right)+.\right)$

$\mathrm{I}_{\mathrm{A}} \sum_{\mathrm{n}=1.7 .19}^{\mathrm{m}} \mathrm{I}_{\mathrm{nM}} \sin \left(\mathrm{n} \omega \mathrm{t}-30^{\circ}\right)+\sum_{\mathrm{n}=5.11 .17}^{\mathrm{m}} \mathrm{I}_{\mathrm{nM}} \sin \left(\mathrm{n} \omega \mathrm{t}+30^{\circ}\right)$ (9)

\section{HARMONIC CURRENTS ANALYSIS}

The phase-shift between both primary star connected and first secondary star- connected winding is $\not=0^{\circ}$ and primary winding and second secondary delta-connected winding is $p=30^{\circ}$ (See Figure (4)). It is important to analyze the behavior of this transformer connection type to determine the transformer primary and secondary harmonic currents cancellation.

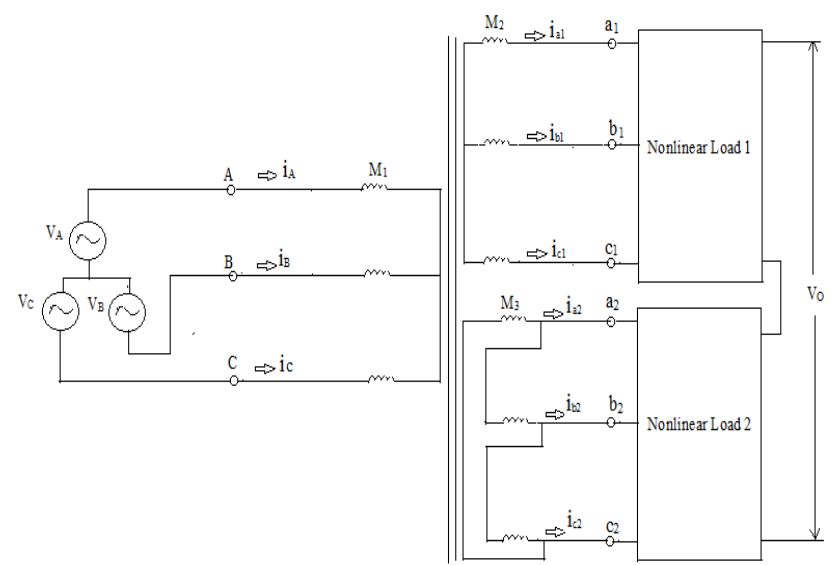

Fig. 4: Three-phase shifting transformer with main primary star winding and two secondary

$$
\begin{aligned}
& \mathrm{i}_{\mathrm{a} 1}=\sum_{\mathrm{n}=1,5,7,11}^{\infty} \mathrm{I}_{\mathrm{nM}} \sin (\mathrm{n} \omega \mathrm{t}) \\
& \mathrm{i}_{\mathrm{a} 2}=\sum_{\mathrm{n}=1,5,7,11}^{\mathrm{m}} \mathrm{I}_{\mathrm{nM}} \sin \left(\mathrm{n}\left(\omega \mathrm{t}+30^{\circ}\right)\right)
\end{aligned}
$$

Phase (A) primary current is equal to $i_{\mathrm{a} 1}+\mathrm{i}_{\mathrm{a} 2}$ after referring to the primary side.

$\mathrm{i}_{\mathrm{A}}=\mathrm{I}_{1 \mathrm{M}} \sin (\omega \mathrm{t})+\mathrm{I}_{11 \mathrm{M}} \sin (11 \omega \mathrm{t})+\mathrm{I}_{13 \mathrm{M}} \sin (13 \omega \mathrm{t})+\mathrm{I}_{23 \mathrm{M}}$ $\sin (23 \omega t)+\ldots \ldots$

It is obvious that all $5^{\text {th }}, 7^{\text {th }}, 17^{\text {th }}$, and $19^{\text {th }}$ harmonic currents has been cancelled.

It is clear from equation (11), that the $11^{\text {th }}$ and $13^{\text {th }}$ harmonics are not eliminated and our decision is to set the connected inverter(s) to eliminate them.

\section{SINGLE INVERTER}

Fig. 5 depicts the three-phase voltage source inverter (VSI) that feeds $9 \mathrm{MW}, 3.3 \mathrm{KV}$ rated induction motor through phase-shifting transformer.

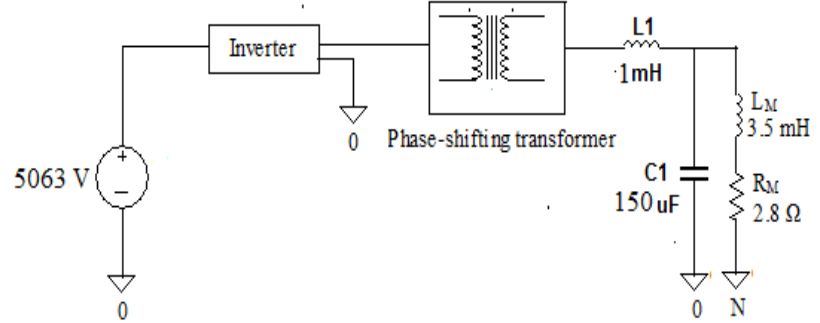

Fig. 5: Three-phase single VSI

The inverter has employed insulated gate bipolar transistors (IGBT) as switching devices. This type of switching devices can operate at the range of voltages (2300-6900) and current available up to 2400A. These switching devices are triggered by SHEPWM scheme. In this inverter, SHEPWM is programmed to eliminate $5^{\text {th }}$, and $7^{\text {th }}$ harmonics. The values of all circuit elements are selected and tested by PSpice to give the optimum THD. The DC supply has been calculated from the following formula considering the load (induction motor) voltage is $3.3 \mathrm{KV} \mathrm{AC}$ rms.

$V_{\mathrm{dc}}=\frac{\sqrt{2}}{\mathrm{MI}} \times 0.73 \times \mathrm{V}_{\mathrm{LL}}$; where VLL is the line-to line voltage, and MI is selected to be 0.98 .

It is worthy to know that $5^{\text {th }}$, and $7^{\text {th }}$ harmonics are not totally cancelled by this way as expected because of the discretization of the switching instances. Table 1 below shows the simulation results for the modulation index (MI) of 0.98 using PSpice and MATLAB software. It is important to know that all three folds harmonics are disappeared due to the property of the SHE-PWM technique. Each value in the Table represents the percentage of the fundamental component of the waveform. The used load is a squirrel cage induction motor with equivalent resistance of (2.8) ohm and inductance of (3.5) $\mathrm{mH}$.

Table 1: Simulation results for single inverter operation with $\mathrm{MI}=0.98$

\begin{tabular}{ccccccc}
\hline Harmonic No. & $\mathbf{5}^{\text {th }}$ & $\mathbf{7}^{\text {th }}$ & $\mathbf{1 1}^{\text {th }}$ & $\mathbf{1 3}^{\text {th }}$ & $\mathbf{1 7}^{\text {th }}$ & $\mathbf{1 9}^{\text {th }}$ \\
\hline PSpice & 0.88 & 0.94 & 3.15 & 21.14 & 5.12 & 2.11 \\
MATLAB & 0.85 & 0.91 & 3.14 & 21.02 & 5.08 & 2.10
\end{tabular}

The value of THD of the load output currents estimated up to (99) harmonics is $7.34 \%$.

\section{PARALLEL OPERATION OF TWO VSI}

From the basic principle of the parallel operation, the output voltage of the system remains the same as with the single inverter previously discussed, but the output current and consequently the total power delivered to the load is doubled. Fig. 6 represents the schematic diagram of two-VSI connected to the load via Phase-shifting transformer. 


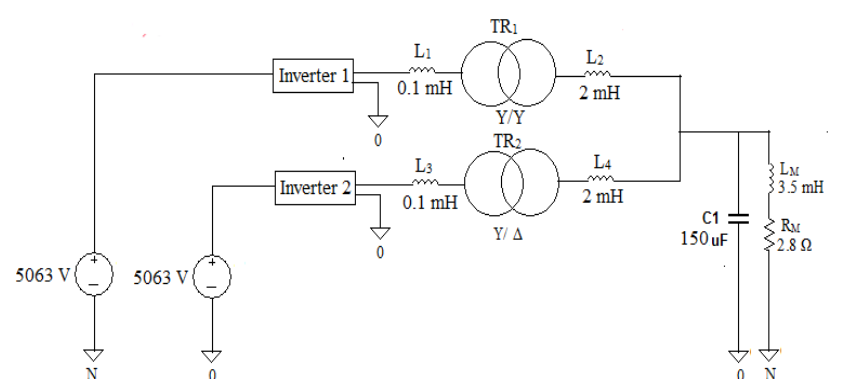

Fig. 6: Two parallel synchronized inverters

In this system, inverter 1 is connected to the star winding of the star to star connected transformer while inverter 2 is connected to the star side of the star to delta connected transformer. This is to eliminate some low order harmonics namely, $5^{\text {th }}, 7^{\text {th }}, 17^{\text {th }}$, and $19^{\text {th }}$ as it is discussed in section IV. Therefore, it is necessary to program first inverter of the topology using traditional SHEPWM to eliminate $7^{\text {th }}$, and $11^{\text {th }}$ harmonics. The second inverter is to be operate as first inverter but the gating pulses are phase-shifted by a pre-calculated value $\mathrm{T} /(2 * 13)$ to eliminate $13^{\text {th }}$ harmonic, where $\mathrm{T}$ is the one cycle period of the sinewave that equals $20 \mathrm{~ms}$. The second inverter is synchronized and set in parallel with the first inverter. The technique here is called modified SHEPWM, in which a small phase-shift angle between the two inverters is created.

A very small circulation current will be generated in the system, but it has not significant impact on the harmonic profile of the entire system. By the combination of phase-shifting transformer and modified SHEPWM technique all low order harmonics up to and including 21th harmonics have been eliminated (or near so in practice). The simulation results for this configuration are shown in the table 2 .

Table 2: Simulation results for two VSI connected to the load via Phase-shifting transformer with $\mathrm{MI}=0.98$.

\begin{tabular}{ccccccc}
\multicolumn{6}{l}{ load via Phase-shifting transformer with $\mathrm{MI}=0.98}$. \\
\hline Harmonic No. & $\mathbf{5}^{\text {th }}$ & ${\mathbf{7}^{\text {th }}}^{\text {th }}$ & $\mathbf{1 1}^{\text {th }}$ & $\mathbf{1 3}^{\text {th }}$ & $\mathbf{1 7}^{\text {th }}$ & $\mathbf{1 9 \text { th }}$ \\
\hline PSpice & 0.94 & 0.43 & 0.23 & 0.88 & 0.98 & 0.98 \\
MATLAB & 0.91 & 0.40 & 0.21 & 0.85 & 0.96 & 0.95 \\
\hline
\end{tabular}

The calculated THD of this topology using PSpice software is $(2.82 \%)$ for harmonics up to (99) harmonics (the maximum capability of PSpice software). Compared with the single inverter, the THD has been reduced significantly from $(7.34 \%)$ to $(2.82 \%)$, and this ensures that all low-order harmonics have been eliminated (or near so in practice) and many high-order harmonics are cancelled because of the LR circuit of the motor which effectively filters out many high-order harmonics.

\section{TRANSFORMERLESS CONNECTION OF THE TWO PARALLEL VSI}

The simulation results of this methodology are shown in Table 3. Below.
Table 3: Simulation results for two-parallel inverters with phase-shifting transformer $(\mathrm{MI}=0.98)$

\begin{tabular}{lcccccc}
\hline Harmonic No. & $\mathbf{5}^{\text {th }}$ & $\mathbf{7}^{\text {th }}$ & $\mathbf{1 1}^{\text {th }}$ & $\mathbf{1 3}^{\text {th }}$ & $\mathbf{1 7}^{\text {th }}$ & $\mathbf{1 9 t h}^{\text {th }}$ \\
\hline PSpice & 3.21 & 0.66 & 0.28 & 0.85 & 4.41 & 1.97 \\
MATLAB & 3.18 & 0.63 & 0.25 & 0.83 & 4.37 & 1.95 \\
\hline
\end{tabular}

The THD estimated by PSpice (up to 99 harmonics) of this configuration is raised to $3.76 \%$ after disconnection of the phase-shifting transformer from the system. It is clear that the $5^{\text {th }}, 17^{\text {th }}$, and $19^{\text {th }}$ harmonics is increased slightly but 7 th, $11^{\text {th }}$, and $13^{\text {th }}$ harmonics remains low due to novel or modified SHEPWM technique.

In brief, the topology of two-parallel inverters with phase-shifting transformer has the better harmonics profile among all other topologies mentioned above [8]. The THD has been reduced to the acceptable limit that is specified by IEEE standard.

\section{CONCLUSIONS}

The modified SHEPWM technique is proved to be very effective in term of cancellation of low-order harmonics and this novel technique reduces the number of trigonometric equations needed for elimination of certain number of harmonics. For instance, we have used three equations instead of four, if traditional SHEPWM is being used, to eliminate three harmonics namely, $7^{\text {th, }} 11^{\text {th }}$, and $13^{\text {th }}$. Therefore a lot of time, efforts, and complicity have been reduced and probable error(s) is/are avoided.

The parallel operation of two inverters employing the modified SHEPWM technique combined with Phase-shifting transformers has contributed to improve the harmonic profile of the system. The THD of the system is reduced to the optimum level which is $2.82 \%$ and consequently the impact of harmonics distortion on the inductive load is tremendously reduced.

Finally, the deviation of the simulation results obtained from PSpice compared with MATLAB is insignificant and this confirms the high accuracy of the results obtained.

\section{REFERENCES}

[1] R. Tajali (2005), Simplifying Harmonic Mitigation for Industrial Plants, Electrical Construction and Maintenance (EC\&M) Magazine.

[2] Sachin K. Jain, and S.N. Singh (2014), Low-Order Dominant Harmonic Estimation Using Adaptive Wavelet Neural Network. IEEE Transactions on Industrial Electronics, vol. 21, No. 1, pp.428-435.

[3] A. Moeini, H. Zhao and S. Wang (2018), A Current-Reference-Based Selective Harmonic Current Mitigation PWM Technique to Improve the Performance of Cascaded H-Bridge Multilevel Active Rectifiers. IEEE Transactions on Industry Electronics, Vol. 65, No. 1, pp. 727-737.

[4] V.G.Agelidis, A. Balouktsis, and I. Balouktssis (2004), On applying a minimization technique to the harmonic elimination PWM control: the bipolar waveform. IEEE power electronics letters,vol.2, issue 2, pp.41-44.

[5] Ramirez, R. W, The FFT Fundamentals and Concepts, Englewood Cliffs, NJ:Prentice-Hall,(1985).

[6] J.R. Wells, Bret M. Nee, P. L. Chapman, and Philip T. Krein (2005), Selective Harmonic Control: A General Problem Formulation and Selected Solutions. IEEE Transactions on Power Electronics, vol. 20, no.6, pp. $1337-1345$. 
[7] H. Zhang, A. von Jouanne, and A. Wallace (2000), Multilevel Inverter Modulation Schemes to Eliminate Common-Mode Voltage. IEEE Transactions on Industry Applications, Vol. 36, No. 6, pp. 1645-1653.

[8] IEEE Recommended practice and requirements for harmonic control in electric power systems, IEEE Std.519-1992, (1993).

\section{AUTHORS PROFILE}

My name is Hussain Bierk, affiliated with College of Engineering, Al-Iraqia University, Baghdad, Iraq, my area of interest is electrical. hbierk@hotmail.com

My good name is Fawzi Mustafa, currently affiliated with College of Engineering, Al-Iraqia University, Baghdad, Iraq

I am Baraa M. Albaker, currently connected with College of Engineering, Al-Iraqia University, Baghdad, Iraq. My area of interest is transformers. 